\title{
ACS: Automatic Converter Synthesis for SoC Bus Protocols
}

\author{
Karin Avnit, Arcot Sowmya, and Jorgen Peddersen \\ The University of New South Wales, \\ Sydney, Australia \\ \{kavnit, sowmya, jorgenp\}@cse.unsw . edu.au
}

\begin{abstract}
In System-on-Chip (SoC) design, pre-designed and preverified modules are often integrated into the system. In the absence of a single interface standard for such modules, "plug-n-play style" integration is not likely, as the modules are often designed to comply with different interface protocols, and a protocol converter is required to mediate between them. ACS is a tool that allows for automatic checking of protocol compatibility and automatic converter synthesis for SoC bus based protocols. It is based on formal foundations and guarantees correct-byconstruction deterministic solutions in VHDL, whenever it is physically possible to mediate between a given pair of protocols.
\end{abstract}

Keywords: System-on-Chip, Protocol converter, Protocol compatibility, Converter synthesis, Bus-based architecture.

\section{Introduction}

Reuse of Intellectual Property (IP) modules has become common practice in chip design. Aimed at accelerating the design phase and increasing system reliability, pre-designed and pre-verified modules are integrated into a single chip. As the integrated modules are often designed by different groups and for different purposes, they typically comply with different interface protocols. For such modules to communicate correctly there is a need for a protocol converter that mediates between them.

In a bus based SoC architecture, the system includes one or more common buses to which all modules interconnect, and all system communication is managed by the bus interface protocols. A general bus-based SoC architecture, including protocol converters for modules is illustrated in Figure 1. For an IP to be integrated into this architecture it needs to either employ an interface protocol that is compatible with the bus protocol, or use a protocol converter.

Automatic Converter Synthesis (ACS) is a tool implementation of methods and algorithms that were developed for provably-correct automatic synthesis of protocol converters for bus protocols of SoCs. It offers instantaneous generation of provably-correct converters for mediation between a given pair of protocols. The methods rely on a dedicated FSM-based formalism and formal definitions 


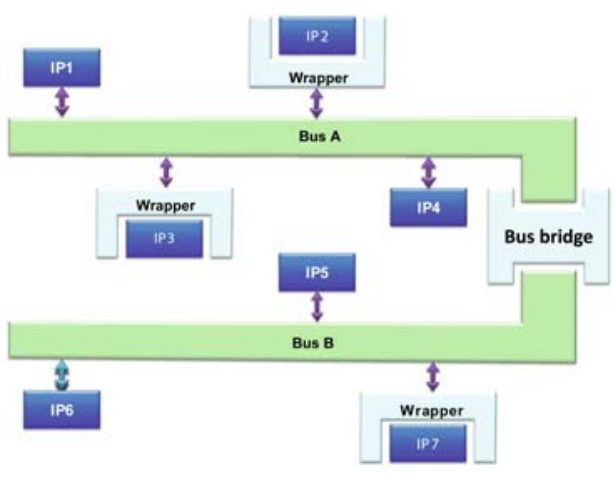

Fig. 1. A typical SoC architecture

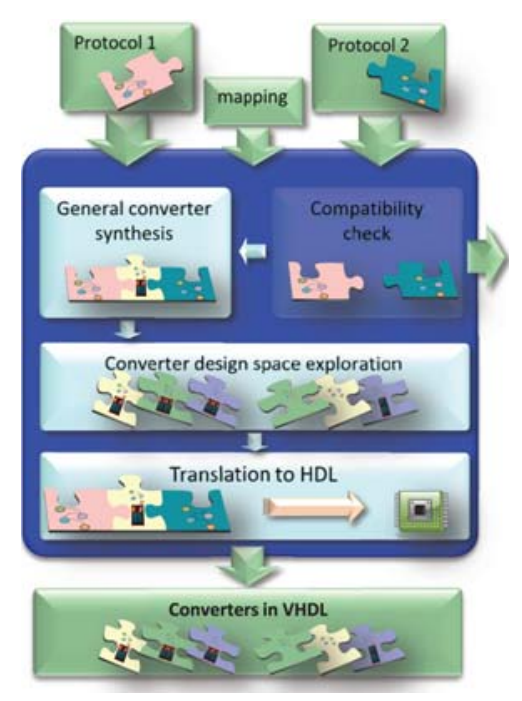

Fig. 2. Tool description

of protocol compatibility and correct protocol conversion [12/3], and the derived algorithm for automatic converter synthesis has proofs of correctness and completeness 2 .

The paper is organized as follows. An overview of the underlying methods and algorithms is presented in section 2, A description of the process flow of the tool is provided in section 3. Experiments with the tool are described in section 4 and the tool status and future work are discussed in section 5 .

\section{The Formal Foundations}

Complete descriptions of the models and methods on which ACS relies are available [12]3. A brief overview is provided here.

The algorithms implemented in ACS are derived from precise formal definition of protocol compatibility and correct protocol conversion that arose from an extensive study of SoC bus protocol characteristics.

The protocol model on which the methods rely distinguishes between control and data channels, and the IP modules are assumed to transfer data items from one to another based on control information, indicating when data items are available on the bus. It is assumed that a data item that is written to the bus may remain valid for more than a single clock cycle and can therefore be read from the bus more than once.

The notion of protocol compatibility, on which the definitions rely, includes three basic conditions. Two protocols are considered to be compatible if and only if:

1. Data is read by one protocol only when written by the other, ensuring that data items are read from the bus only when the bus is guaranteed to hold a valid value. 
2. A given data item is read as distinct exactly once. This guarantees a mutual understanding between the communicating parties of the amount of data that is transferred and prevents loss of data and incorrect data duplication.

3. No deadlocks can occur and livelocks can always be avoided, ensuring that every transfer can terminate in a finite number of steps.

A protocol converter is defined as an FSM with bounded counters and finite buffers. The buffers store data items that were written to the bus by one protocol until the other protocol is ready to read them. The general notion of correct protocol conversion includes two conditions. A converter mediating between two protocols is correct if and only if:

1. The converter is compatible with each of the protocols, and thereby complies with the three conditions above.

2. The converter remains neutral in the conversation between the protocols. It transfers all and only data items that were provided to it by the protocols.

The definition of correct conversion was derived from these notions, and the algorithm for converter synthesis was designed to guarantee that the converters produced comply with this definition. Typically, more than one converter exists that guarantees correct mediation between protocols. In search of these converters, the first step in automatic synthesis of protocol converters produces a machine referred to as the Most General Correct Converter ( $M G C^{2}$ ), which is a nondeterministic converter that describes all possible behaviors a of a correct converter and restricts the design space for correct converters. The $M G C^{2}$ is computed using an iterative algorithm that starts with the construction of an initial converter that allows every possible sequence of transitions in both protocols (whether correct or not). This is achieved in two simple steps - the computation of the cross product of the protocols, and an inversion of channel actions of the protocols (according to a predefined inversion function). The converter synthesis algorithm then iteratively restricts the initial converter to only correct behaviors, until a fixed point is reached, in which none of the behaviors enabled by the converter causes a violation of the definition for correct conversion.

As the produced $M G C^{2}$ is potentially non-deterministic, an algorithm for design space exploration of the $M G C^{2}$ was also developed [3]. The algorithm extracts deterministic converters out of the $M G C^{2}$, that are guaranteed to comply with the definition of correct protocol conversion. This is achieved by the identification of input control conditions for which more than a single behavior is enabled, and the construction of sub-converters in which a priori choice is taken to include only one behavior under the identified conditions.

\section{ACS Process Flow}

The input to the tool consists of a pair of protocol models and mapping information. A protocol is specified as a directed graph in the DOT format [4. The mapping information indicates the relation between the data channels of the two 
protocols (an output data channel of one protocol is mapped to each input data channel of the other protocol). The process flow of the tool, as described in this section, is illustrated in Figure 2

After loading the input information, in which a syntax check is performed, and setting some basic parameters (the size of buffers to be used in the converter), the user can choose to perform an analysis, in which it is checked if a converter exists for the pair of protocols under the specified parameters. Based on the construction of the $M G C^{2}$, a partial analysis option reports on an upper bound for the number of unique converters that exist, while a full analysis reports on the exact number of converters that exist and produces a report on the number of states and transitions in each of the potential converters. A model of the $M G C^{2}$ is also provided in the DOT format.

The next step after the analysis of the $M G C^{2}$ is the converter synthesis. If specified by the user, the tool can export all converters into files in both the DOT format and VHDL, enabling instant simulation, comparison between the different converters, and direct integration into the SoC design.

For a given pair of protocols, the output of the tool is a list of deterministic, provably-correct converters in VHDL, the number of which depends on the given protocols and settings. A system designer at this point may choose which of the converters and integrate it into the system with the use of any standard hardware design IDE application (such as Xilinx ISE [5]). Comparisons between the different converters produced by the tool can be made based on the number of states and transitions in the converters, the size of buffers that each converter uses (a report of these parameters is produced by the tool), or if other criteria for comparisons are desired, the designer may wish to run simulations of the converters in standard hardware simulation tools and examine the converter performance as done with any VHDL design.

\section{Experiments}

The PC-based tool was used to generate converters for a number of simple examples, as well as full scale commercial protocols such as the AMBA protocol family 6778 and the Open Core Protocol (OCP) 9]. Results of experiments with commercial protocols are reported in Table 1 . The table shows the number of states and transitions in each $M G C^{2}$, the number of converters found relative to the number of different combinations of choices that exist in the $M G C^{2}$ (\# column), and the execution time on a standard PC (Pentium 4 CPU 3.2GHZ, $2 \mathrm{~GB}$ of RAM). It also lists the number of states and transitions in the minimal converter found in each experiment and the improvement it shows relative to the $M G C^{2}$.

The experiments illustrate a number of points regarding automatic converter synthesis. First, the significant difference between the potential number of choice combinations and the number of correct converters found implies that the likelihood of a successful manual or random search for a deterministic converter is low and a systematic search is needed. Taking for example the converters between 
Table 1. Experimental results

\begin{tabular}{|c|c|c|c|c|c|c|c|c|c|c|}
\hline \multirow{2}{*}{ Initiator } & \multirow{2}{*}{ Reactor } & \multirow{2}{*}{ Buffer } & \multicolumn{2}{|c|}{$M G C^{2}$} & \multirow{2}{*}{ \# } & \multirow{2}{*}{ Time } & \multicolumn{2}{|c|}{ Min } & \multicolumn{2}{|c|}{ Improvement } \\
\hline & & & States & Trans & & & States & Trans & States & Trans \\
\hline \multirow{3}{*}{ ASB } & \multirow{3}{*}{ APB } & 1 & 0 & 0 & - & $<1$ sec & - & - & - & - \\
\hline & & 2 & 10 & 19 & $4 / 96$ & $<1$ sec & 8 & 13 & $20 \%$ & $\mathbf{3 1} \%$ \\
\hline & & 5 & 10 & 19 & $9 / 96$ & $2 \mathrm{sec}$ & 8 & 13 & $20 \%$ & $31 \%$ \\
\hline \multirow{2}{*}{ OCP } & \multirow{2}{*}{ APB } & 2 & 4 & 15 & $4 / 1,024$ & $3 \mathrm{sec}$ & 2 & 3 & $\mathbf{5 0} \%$ & $80 \%$ \\
\hline & & 10 & 4 & 15 & $4 / 1,024$ & $5 \mathrm{sec}$ & 2 & 3 & $\mathbf{5 0} \%$ & $80 \%$ \\
\hline $\mathrm{OCP}$ & ASB & 2 & 14 & 32 & $4 / 4,096$ & $1 \mathrm{~min}$ & 7 & 10 & $56 \%$ & $\mathbf{7 5} \%$ \\
\hline (READ) & (READ) & 10 & 14 & 32 & $4 / 4,096$ & $1 \mathrm{~min}$ & 7 & 10 & $56 \%$ & $75 \%$ \\
\hline $\mathrm{OCP}$ & $\mathrm{ASB}$ & 2 & 16 & 40 & $1102 / 65,536$ & $15 \mathrm{~min}$ & 7 & 10 & $56 \%$ & $\mathbf{7 5} \%$ \\
\hline (WRITE) & (WRITE) & 10 & 16 & 40 & $1102 / 65,536$ & $30 \mathrm{~min}$ & 7 & 10 & $\mathbf{5 6} \%$ & $\mathbf{7 5} \%$ \\
\hline
\end{tabular}

an OCP master and an APB slave, the design space of the converters include 1024 different combinations of choices between behaviors, and only 4 of these combinations yield valid unique converters. Second, the table shows that there can be dramatic differences between different converters for the same pair of protocols, by showing that the minimal converter found is significantly smaller than the $M G C^{2}$. In the example of OCP to APB converters, the $M G C^{2}$ has 4 states and 14 transitions, but the minimal converter has only 2 states and 3 transitions, which is a significant difference. In this case the measure for comparison was the physical size of the converters (number of states and transitions) but this comparison can be made for any other meaningful measure that can be estimated, such as buffer utilization, power consumption etc. The differences between converters emphasize the advantage of using automatic synthesis in improving system performance, and this difference appears to grow with the complexity of the protocols. This can be attributed to the flexibility of complex protocols, which allow for greater choices for the communicating party, and in this case the converter.

Converters that were produced by the tool were successfully synthesized to FPGAs and simulated with the use of standard commercial tools for hardware design (Xilinx ISE, Modelsim and Synplify simulators).

Note that it is not always possible to generate a converter for any pair of protocols or for any size of buffers. This limitation has to do with the characteristics of the protocols involved. For example, an AMBA2 APB master follows a very simple protocol that does not include any wait states and expects, for example, that any "read" request be serviced within one clock cycle. Such a protocol cannot be correctly converted to more complex slaves such as ASB or OCP, since these slaves do not guarantee an immediate response and may try and force wait states on the master. No converter can overcome such differences and guarantee correct conversion for any legal behavior of the protocols. In such cases where it is not possible to generate a converter the tool will report that the $M G C^{2}$ could not be produced. 


\section{$5 \quad$ Status and Future Work}

ACS was implemented as a part of ongoing research in formal methods for hardware design automation, and as such it is evolving continuously, following advances in the research. The tool currently supports automatic converter synthesis for protocols with unidirectional data channels and matching data bus widths between protocols, although the algorithms for mismatched data widths have been already developed [2] and will soon be integrated into the tool. Other extensions that will be integrated into the tool in the coming months include automatic compatibility check 2] with counter examples on failure, and buffer size suggestions.

\section{References}

1. Avnit, K., D'Silva, V., Sowmya, A., Ramesh, S., Parameswaran, S.: A formal approach to the protocol converter problem. In: DATE 2008, March 10-14, pp. 294-299 (2008), doi:10.1109/DATE.2008.4484695

2. Avnit, K., D'Silva, V., Sowmya, A., Ramesh, S., Parameswaran, S.: Provably correct on-chip communication: A formal approach to automatic protocol converter synthesis. ACM Trans. Des. Autom. Electron. Syst. 14(2), 1-41 (2009)

3. Avnit, K., Sowmya, A.: A formal approach to design space exploration of protocol converters. In: Design, Automation \& Test in Europe Conference \& Exhibition. DATE 2009, April 2009, pp. 129-134 (2009)

4. Gansner, E., North, S.C.: Drawing graphs with dot. AT\&T Bell Laboratories, Murray Hill (2002)

5. Xilinx, I.: Software Manuals and Help, http://www.xilinx.com/support/documentation/index.htm

6. ARM: AMBA2 specification IHI 0011A (May 1999), http://www.arm.com/products/solutions/AMBAHomePage.html

7. ARM: AMBA3 Advanced Peripheral Bus specification IHI 0024B (October 2004), http://www . arm. com/products/solutions/AMBAHomePage.html

8. ARM: AMBA3 AHB-lite specification IHI 0033A (June 2006), http://www.arm.com/products/solutions/AMBAHomePage.html

9. OCPIP: Open Core Protocol International Partnership (2005), http://www.ocpip.org 